\title{
Le rôle des agents français dans la constitution
} batave de 1798

The role of French agents in the Batavian Constitution of 1798.

\section{Annie Jourdan}

\section{(2) OpenEdition \\ 1 Journals}

Édition électronique

URL : https://journals.openedition.org/ahrf/11367

DOI : 10.4000/ahrf. 11367

ISSN : 1952-403X

\section{Éditeur :}

Armand Colin, Société des études robespierristes

\section{Édition imprimée}

Date de publication : 1 mars 2008

Pagination : 99-119

ISSN : 0003-4436

\section{Référence électronique}

Annie Jourdan, "Le rôle des agents français dans la constitution batave de 1798 », Annales historiques de la Révolution française [En ligne], 351 | janvier-mars 2008, mis en ligne le 01 avril 2011, consulté le 03 août 2021. URL : http://journals.openedition.org/ahrf/11367 ; DOI : https://doi.org/10.4000/ahrf. 11367 


\title{
LE RÔLE DES AGENTS FRANÇAIS \\ DANS LA CONSTITUTION BATAVE DE 1798
}

\section{Annie JOURDAN}

\begin{abstract}
La Révolution batave est un domaine encore en friche. Non seulement on ignore les grandes réformes entreprises ou les acquis réels de la période, mais l'interprétation des événements euxmêmes laisse à désirer. Parmi ceux-ci le rôle précis des agents français dans l'élaboration de la Constitution batave de 1798, la première à avoir été effectivement introduite et à avoir fonctionné durant trois ans. De même, on ignorait jusqu'à présent les diverses raisons qui ont mené à la chute des radicaux, pères fondateurs de cette constitution. L'article s'interroge donc sur ces deux aspects, analysant dans le détail les relations complexes entre agents français et révolutionnaires bataves et recherchant les divers éléments qui ont pu causer la chute et le discrédit du gouvernement radical de janvier 1798. Pour conclure, quelques caractéristiques de la constitution et de la déclaration sont évoquées, lesquelles témoignent de l'originalité de ces deux créations, qui sont le résultat d'un riche dialogue entre Français et Bataves : dialogue donc, voire émulation et non point servile imitation ou impérieuse contrainte. C'est dire que ce texte nuance également l'interprétation traditionnelle entre République mère et républiques sœurs.
\end{abstract}

Mots-clés : Constitution batave, Directoire, Charles Delacroix, Brahaïn Ducange, Déclaration des droits, Radicaux bataves, républiques sœurs.

Traiter de la Révolution batave n'est pas chose facile, si complexes sont les enjeux qui vont de pair, et d'autant qu'elle comprend deux séquences : la révolution des patriotes de 1781-1787 et la révolution batave 
de 1795-1806. Qui plus est, la période a été relativement peu étudiée par l'historiographie néerlandaise. Et quand elle l'a été, cela a été le fait de conservateurs ou d'orangistes, et plus rarement de sympathisants, ce qui, on s'en doute, fausse d'emblée l'interprétation ou l'oriente dans un sens univoque. Il n'y a pas eu non plus dans le pays de tradition scientifique socialiste ou marxistc comme en France, qui aurait pu mettre en valeur les avancées sociales et économiques ou les initiatives populaires. Nombre de facettes ont donc été passées sous silence et le sont encore, tandis que d'autres étaient survalorisées'. Cela vaut tout particulièrement pour ce qui est de l'influence française sur les réalisations politiques et juridiques. Le premier grand historien de la période, Herman Colenbrander assimile ainsi les patriotes des années 1780-1800 à des marionnettes dont les grandes puissances - la France, en particulier - tirent les ficelles. Son successeur Pieter Geyl, qui conteste cette interprétation, la rejoint en un sens quand il se refuse à déceler un quelconque radicalisme parmi ses compatriotes. De là son peu d'intérêt pour les hommes du coup d'État (radical) du 22 janvier 1798, qui ne sauraient avoir agi de façon autonome. Ce serait la France qui, selon lui, gouverne désormais par le biais de ses agents ou ministres plénipotentiaires. Influencé par les thèses de Robert Palmer sur la révolution démocratique qui secoue le monde Atlantique dans les années 1780, l'historien libéral Cornelis de Wit restitue leur originalité aux événements et voit une continuité évidente entre les patriotes des années 1780 et les Bataves des années 1790. Et de fait, ce sont bien souvent les mêmes hommes ${ }^{2}$. Mais il ne résout pas pour autant les problèmes et en crée un à l'inverse, quand il dote les protagonistes de l'étiquette duelle et simpliste : aristocratie versus démocratie. Simon Schama qui prend la relève en 1977, n'abandonne pas explicitement cette interprétation et malgré des recherches d'archives très sérieuses, se soucie moins de proposer de nouvelles perspectives que de décrire, fort bien du reste, les événements qui mènent à la démocratie. Comme de Wit, il lit ces années-là comme devant nécessairement aboutir à 1848 , date de l'introduction aux Pays-Bas d'une constitution libérale. Mais c'est dire que l'un et l'autre confondent révolution patriote et révolution batave, d'une part, et démocratie, républicanisme et libéralisme, d'autre part, tandis qu'ils ont du mal à distingucr entre radicaux ou entre factions et jugent les années révolutionnaires à l'aune de ce qui ne sera atteint qu'en 1848 .

(1) Sur l'historiographie, Nick VAN SAS, De metamorfose van Nederland. Van oude orde naar moderniteit, 1750-1900. Amsterdam, Amsterdam University Press, 2004.

(2) On trouvera un article en français de Cornelis DE WIT, “La République batave, 1795-1805 * dans Occupants, Occupés, éd. Devleeshouder, Bruxelles, 1969, p. 141-164. Herman Colfnbrander, De Bataafsche Republiek, Amsterdam, 1908. 
Depuis, les recherches se sont heureusement multipliées. La révolution patriote a fait l'objet de nombreuses études et a été remarquablement valorisée. Les patriotes en exil ont reconquis leur identité ; les relations américano-néerlandaises sont mieux connues, tout comme les divers projets de constitution, les réformes judiciaires ou la politique fiscale et financière. Plusieurs travaux abordent aussi dans leurs détails la révolution au village, en ville ou en province, d'autres se penchent sur la sociabilité politique, le patriotisme et le républicanisme ; d'autres encore s'interrogent sur la participation féminine dans le grand œuvre révolutionnaire ou sur la politique culturelle et artistique. En bref, ces ouvrages nuancent l'approche traditionnelle et montrent bien l'originalité de la période, mais ils demeurent fragmentaires. Jusqu'ici fait défaut en effet une synthèse à la fois globale et analytique sur l'histoire politique, juridique et culturelle de la Révolution batave et sur ses projets constitutionnels, déclaratoires et judiciaires. Autant la révolution patriote est désormais bien connue, autant la Révolution batave reste malgré tout le parent pauvre de l'histoire des révolutions d'Occident'. L'historiographie néerlandaise a été en réalité influencée par celle qui s'écrivait en France et qui minorait les événements étrangers au profit de 1789 ou de 1793 . Et ce n'était pas pour lui déplaire, puisque si le pays avait été sous l'emprise de la "Grande Nation », la Hollande en ressortait absoute de tous les " crimes " révolutionnaires. Jamais, elle n'aurait de son plein gré exilé le prince d'Orange ou rêvé d'une démocratie populaire. Limpérialisme napoléonien entre-temps avait en effet discrédité les avancées réellement révolutionnaires et faussé en somme et pour longtemps l'interprétation de la Révolution bataves.

Au vrai, l'étude des révolutions occidentales devrait permettre non seulement de mieux les connaître en particulier, mais aussi de renouveler l'interprétation de la Grande Révolution de France. Ainsi, en dépit d'une tradition tenace qui consiste à affirmer que les républiques sœurs se sont alignées par goût ou par force sur le modèle par excellence, une étude pointilliste de la Révolution batave démontre tout à la fois que Paris n'a pas toujours voulu imposer sa loi et que les législateurs hollandais avaient des idées précises - mais variées - sur le régime qu'ils souhaitaient instaurer. Entre janvier 1795, quand arrivent en Hollande les armées franco-

(3) Nous avons entrepris telle histoire globale, qui traite tout à la fois du politique, du juridique et du culturel dans une perspective comparatiste. Louvrage devrait paraitre prochainement.

(4) À ce sujet, voir notre introduction au numéro spécial des $A H R F$ sur la révolution batave, $\mathrm{n}^{\circ} 4$, 2001, p. 1-23. Ft Willem Fristioff \& Jonst RostNDAAL, "La révolution régénérée : nouvelles approches et nouveltes images de la révolution nécrlandaise » dans L.'image de la Révolution française, éd. M. Vovelle, Pcrgamon Press, I, 1990, p. 543-561.

(5) $\dot{A}$ tel point qu'au $X X^{\circ}$ siècle, il y a eu des historiens pour interpréter la période comme étant identique à celle de la collaboration allemande. C'est contre cette historiographie tendancicuse que Pieter Geyl a écrit sa "révolution batave », Pieter GeYL, La Révolution batave (1783-1798), Société des Études robespierristes, Paris, 1971. 
hollandaises, lesquelles " libèrent " le pays du " joug stadhouderien ", et août 1797, quand le peuple rejette le texte constitutionnel rédigé durant les quinze mois précédents, bien des discussions ont opposé les membres de la Convention batave et maints incidents ont retardé l'achèvement de la révolution, sans que la France n'intervienne. Trois ans après leur « révolution de velours » et nombre de palabres, les Bataves sont enfin parvenus à terminer un texte, qui ne fait donc pas l'unanimité dans le plat pays'. Trois ans durant lesquels la France a laissé faire et a seulement encouragé Noël, son ministre à La Haye, de convaincre la « république sœur » d'adopter l'unité et l'indivisibilité. Et ce n'est qu'après le rejet du texte que Paris s'impatiente vraiment. Sans existence politique réelle, la république sœur n'est en effet d'aucun apport pour le Directoire qui revendique un appui financier, militaire et naval de la part d'une alliée, laquelle lui est redevable de sa libération. C'est sur ce fond d'atermoiements jugés insupportables à la fois par le Directoire français et les radicaux bataves que vont se précipiter les événements. Au lendemain du 18 fructidor an V, persuadés que leur république est dans une impasse, mais conscients que le fruit est mûr, les radicaux bataves ${ }^{7}$ décident de recourir à la force pour imposer une constitution et sauver leur révolution. C'est de cet épisode mouvementé qu'il sera question ici.

\section{Les intrigues de Ducange et la réalisation de la Constitution de 1798}

Parmi les nombreux mystères que recèle la révolution batave ${ }^{\natural}$, un des plus intéressants est sans nul doute le processus de réalisation de la constitution et le rôle qu'y ont joué les représentants de la Francey.

(6) Les rctards sont dus aux divisions intenses entre fédéralistes et unitaristes d'unc part et entre radicaux et modérés, d'autre part. Cf. mon livre à paraître sur la Révolution batave.

(7) Voir mon Working Paper, "Amsterdam en révolution. Un jacobinisme batave ? ". Sur www.europesestudies.nl, pour une première approche des divers partis bataves - qui diffèrent fort de leurs homologues français ou du moins de l'image que l'on a d'eux. Car rien ne dit que de nouvelles études ne nuancent cette image. Pour exemple, dans LQ révolution des droits de lhomme, Gallimard, 1989, Marcel GAUCHET montre bien tout ce qui oppose les prétendus Girondins entre eux, de même que les divergences entre Jacobins.

(8) Rappelons les ouvrages accessibles au public non néerlandophone : P. GeYL, La révolution batave (1783-1798), Paris, 1971. Geyl n'aborde pas les coups d'État de 1798, car il les perçoit comme étant le fait des Français, tandis qu'il ne croit donc pas en un radicalisme batave. Son étude se termine au 25 janvier 1798. S. Schama, Patriots and Liberators. Revolution in the Netherlands (1780-1813), Knopf, New York, 1977. Schama se contredit : tantôt il souligne la modération néerlandaise ; tantôt il montre le radicalisme à l'cuvre, sans tirer les conclusions qui s'imposent, parce qu'il prend pour contrepoint le radicalisme français. Parmi les études récentes consacrécs à la révolution batave, aucun auteur ne s'interroge par ailleurs sur le pourquoi de la chute des radicaux, comme si celle-ci allait de soi. Depuis la parution du livre de Schama, les synthèses de Godechot et de Palmer sont quelque peu dépassées, même si elles contiennent des informations encore utiles. Qui plus est, elles se focalisent trop sur les ressemblances ct trop peu sur les divergences entre pays en révolution. Et surtout, elles tiennent peu compte du contexte et de la culture dans lesquels s'inscrivent ces révolutions à la fois si proches et si dissemblables, ce qui fausse à terme une juste appréhension du phénomène.

(9) Lhistoriographie néerlandaise est divisée sur ce point, mais, dans l'ensemble, historiens et juristes s'accordent pour lire une influence française indéniable dans la Constitution et la Déclaration. 
Fin décembre 1797, en effet, Charles Delacroix, ci-devant ministre des Affaires extérieures, est envoyé en Hollande où il va remplacer Noël, ministre à La Haye depuis septembre 1795. Le précède de quelques jours Brahaïn Ducange, son secrétaire particulier pour une mission de première importance : faire accepter une constitution au peuple batave, qui vient de rejeter un premier projet en août 1797. Cette nomination - à laquelle s'ajoute celle du général Joubert, qui se substitue à Beurnonville - est le résultat d'intrigues menées à Paris par des agents des radicaux. Parmi ces derniers, Pieter Vreede, fabricant de draps, qui fonctionne comme le chef officieux du « parti »; Wybo Fijnje, intellectuel et journaliste ; Stefanus van Langen, fabricant de draps ; Johan Fokker, théologien et philosophe ; Willem Ockerse, pasteur et Henricus Midderigh, marchand de vin. Ce sont là les personnalités éminentes du parti radical à l'origine du coup d'État du 22 janvier et ce sont elles aussi qui doivent s'assurer du soutien de Ducange et par suite de celui de Delacroix. Assurés du dévouement de Ducange, les radicaux - " ou ceux que l'on nomme les ultra-révolutionnaires », ainsi que l'écrit Delacroix lui-même - le sont sans nul doute. C'est déjà ce qu'affirme le 21 décembre 1797 Eberstein, espion du parti radical de séjour à Paris à Bode, un révolutionnaire notoire d'Amsterdam"

Brahain Ducange, personnage énigmatique, comme il y en eut tant à l'époque, a derrière lui une carrière pour le moins mouvementée. Collaborateur à la Gazette de Leyde de 1776 à 1792, il a dû ensuite se réfugier à Paris et a joué le rôle d'intermédiaire entre les réfugiés hollandais et les Jacobins. À plusieurs reprises, il est leur porte-parole au club de la rue Saint-Honoré où il parle en faveur de la guerre et de la libération des Provinces-Unies. Quand Johan Valckenaer, un radical peu ordinaire, entré dans la postérité sous l'étiquette de jacobin", décide de créer son journal Le Batave, Ducange en devient un des rédacteurs, jusqu'en août 1793, date de son mariage avec une femme aisée, ce qui lui permet de se retirer du journalisme. Ses activités politiques ne s'en ressentent pas outre mesure. On le retrouve membre du Comité des recherches et des liquidations, notamment des biens des banquiers suspects ou incarcérés. En avril 1794, il est exclu des Jacobins en raison de ses liaisons avec des étrangers, notamment le banquier Abbema, lui aussi suspecté et emprisonné. Ducange

En vérité, pour parvenir à des conclusions fiables, il faut étudier point par point les divers projets et textes de loi, tels qu'ils ont été élaborés depuis 1785-1787. Pour un aperçu des textes de l'ćpoque, notre site à l'Université d'Amsterdam : http://home.medewerker.uva.nl/a.r.m.jourdan/ documents

(10) Lconard DF: GoU, De Staatsregeling van 1798. Bronnen tot de totstandkoming, La Haye, 1988, 2 vol. Sera abrégé De Gou, 1798,1, p. 589.

(11) Johan Valckenaer, homme d'influence auprès de patriotes de tous bords est radical, pour ce qui est des moyens. Sur les fins, il dit lui-même en 1801 s'accorder tout à fait avec Schimmelpenninck, et, durant son exil en France, il fréquente non les « lêtes exaltées » du Comité révolutionnaire batave, mais les régents patriotes. Herman COLENBRANDFr, Gedenkstukken der Algemeene geschiedenis van Nederland, 21 vol., La Haye, 1905-1922, 1801, III, p. 718. Sera abrégć GS. Joost ROSENDAAL, Bataven ! Nederlandse vluchtelingen in Frankrijk, 1787-1795, Vantilt, Tilbourg, 2003, entre autres p. 583. 
parvient à se disculper et, en juin 1794, se retrouve à nouveau liquidateur de biens. Après un silence de quelques années, où il semblerait avoir escroqué plusieurs personnes, le revoilà sur scène en décembre 1797 , en tant qu'agent de Barras' ${ }^{12}$.

Avant même le départ de Ducange pour La Haye, Eberstein a donc pris contact avec lui et s'est assuré qu'il défendrait le point de vue des patriotes avancés. Ceux-ci devront « lui faire la plus grande confiance et lui donner cent ducats d'abord, avec une promesse de mille ducats supplémentaires, s'il parvient à convaincre Delacroix d'opter pour le patriotisme ». Lagent des radicaux à Paris l'a mis en contact avec Eyckenbroek, son comparse dans l'intrigue nouée depuis plusieurs mois pour s'assurer du rappel de Noël et de Beurnonville. Dès fin décembre 1797, Ducange a ainsi rencontré divers radicaux bataves et peut assurer à Delacroix qu'il s'agit là d'hommes dociles, honnêtes et de bonne foi, avec qui le ministre français trouvera facilement un terrain d'entente. Le 2 janvier suivant, pourtant, Delacroix n'a pas encore contacté leurs partisans. Ceux-ci s'inquiètent : «ne serait-il pas monté contre les quarante-trois? ». Le 3, Ockerse et ses amis prennent l'initiative : «ils vont aller le voir sans aucune explication ». La rencontre a dû se faire le 4 , car, dès le lendemain, Ducange informe le ministre de la France que les patriotes "dressent sur papier les principaux articles en litige et paraissent se rapprocher de ce que l'on désire d'eux ". Parmi ceux-ci, la crème de la crème de la faction radicale : notamment Van Langen et Ockerse. Le 6, ils ont déjà rédigé un plan provisoire, qui sera terminé le 8. Ducange s'engage à le traduire et à le porter au ministre. Et surtout, il prétend qu'il y a " peu de différence entre ce qu'ils ont rédigé et ce que veut la France ». Le 9 janvier au matin, Ducange envoie les premiers articles au ministre : un «brouillon constitutionnaire » et il l'avertit que le «parti stadhouderien » est lui aussi entré en lice. Il se fait fort du reste de discréditer le dit parti. Le 11, il réaffirme que l'on « $s$ 'en tiendrait aux articles que le gouvernement français désire voir adopter. C'est un point convenu ». Quatre jours plus tard, Ockerse écrit à Samuel Wiselius, un " démocrate " radical, que l'agent de Barras les tient au courant des intentions du ministre. Lui et ses amis se sont déjà accordés avec ce dernier sur le fond. Reste à formuler les principes, ce qu'ils entendent faire d'ici deux jours. Jusque-là, les Bataves n'ont donc reçu aucun projet français quelconque, mais élaborent un plan, inspiré du manifeste des 43 , paru le 12

(12) Sur ces événements et intrigues, voir aussi l'interprétation de DE Gov, 1798, 1, p. IX-LXVII. Pour un résumé (négatif) de la vie de Ducange, Authentique bijlagen tot de gebeurtenissen van den 12 juni 1798, Leyde, $1798, n^{\circ} 7$, p. 4-10. 
décembre précédent ${ }^{13}$, dont ils élimineront les articles qui ont par trop choqué le Directoire français ${ }^{14}$.

Delacroix ćtait pourtant parti de Paris, avec dans ses bagages, un " projet de constitution » pour la République batave ", qui ne saurait être que celui de Daunou, ainsi que le suggère l'intitulé. Pour le reste, il n'avait point d'instructions précises sur les hommes à aborder et pas non plus de "symbole constitutionnel », comme se sont plu à le croire certains historiens $^{15}$. En réalité, jusqu'au 9 janvier au soir, à savoir le jour même de la réception de «l'aperçu des différents points de la Constitution » conçu par les radicaux, dont la traduction lui a été remise par Ducange, Delacroix n'a énoncé aucune opinion sur les bases précises où devait reposer la constitution. Ce soir-là, devant les modérés de la Commission des relations extérieures, qui lui demandent de leur communiquer ses idées sur ces fameuses bases, le ministre avoue que le Directoire français verrait avec plaisir : « une division du corps législatif en deux chambres; un pouvoir exécutif de cinq membres ; sous lui des ministres et non des commissions ; la séparation et l'indépendance absolue du pouvoir législatif et exécutif du pouvoir judiciaire ; point de haute cour nationale permanente $[. .$.$] et point de$ sociétés populaires délibérantes ${ }^{16}$. Ce seraient là les seules bases à imposer aux législateurs bataves, car, à cette date, Delacroix n'a donc toujours pas élaboré ce qu'il appellera plus tard son « symbole constitutionnel ». Et si le 10 janvier, il est en mesure de faire parvenir à Talleyrand un premier projet, tout semble indiquer que c'est le « brouillon constitutionnaire » des radicaux, reçu la veille.

En vérité, ce symbole, ce sont tout simplement les «points constitutionnels convenus ", fruit des discussions entre les radicaux et Delacroix d'après les principes du manifeste des 43 . Entrepris dès le début janvier, traduits par Ducange et successivement corrigés ou améliorés par le ministre

(13) Sur Ducange, J. RosendAAL, op. cit. p. $355-58$; p. 408-409. Ces données proviennent des sources suivantes : AN, AFIII, 70, dossier 283. Pour une grande part, reproduit dans DE Gou, 1798, I, p. 589-612 et II, p. 350-413 (diverses lettres de Ducange, Ockerse, Delacroix). GS, II, p. 580-584. Notons que Delacroix avoue ouvertement prendre pour base les " excellents principes " du manifeste des 43, mais en élimine ce qu'il appelle « la lie ultra-révolutionnaire " (DE GOU, 1798, I, p. 611, lettre à Talleyrand du 10 janvier 1798). Nous ne mentionnerons plus ces références.

(14) GS., II, p. 581-582. Eberstein écrit ainsi aux radicaux que « l'article 3 ; l'article sur le veto et celui qui suit $"$ ont été beaucoup critiqués à Paris. Voir note précédente sur la réaction de Delacroix. DE GOU, 1798, I, p. 611-613.

(15) AE Mémoires et documents, vol.71. Voir les instructions à Delacroix du 12 frimaire an VI : « Le premier point de la mission importante confiéc au citoyen Charles Delacroix sera done de concourir à accélérer la discussion de cet acte constitutionnel et à faire qu'il soit approuvé de la Convention nationale». Delacroix était déjà averti des obstacles que posait la commission des relations extérieures batave, mais Talleyrand ne lui avait donné aucune indication sur les hommes à aborder. DE Gou, qui a pourtant fort bien étudié la période continue de croire en l'existence d'un symbole constitutionnel, distinct des points constitutionnels convenus (op. cit. p. XXXV-XXXVI). Schama ne dit pas autre chose, op. cit., p. 300-303, de même que GeyL, op. cit., p. 366-375.

(16) Les points mentionnés par Delacroix à la commission des relations extérieures sont ccux auxqucls tenait le Directoire parisien. DE Gou, 1798, II, p. 380. Sur Delacroix et les principes développés le 9 janvier, DE GOU, 1798, I, p. 611-613. 
de la France, les points constitutionnels convenus, qu'Ockerse qualifiera justement de "symbole de notre indépendance ", sont acceptés le 21 janvier pour base de leurs travaux par 49 députés - mais aussi par Delacroix. Le 25, trois jours après le coup d'État dirigé par les généraux Daendels et Joubert, un rapport détaillé sur les points convenus est fait à l'Assemblée par le député Rant. S'y décèlent clairement quelques différences avec le premier projet, qui vont dans le sens d'une plus grande tolérance vis-à-vis des parents d'émigrés, des ci-devant nobles et des fonctionnaires de l'Ancien Régime - ce qui pourrait indiquer que les articles répressifs émanaient plus particulièrement du ministre de la France et ne répondaient pas aux aspirations de la majorité du comité de Constitution ${ }^{17}$.

Pendant ce temps, Ducange et ses comparses avaient préparé le fameux coup d'État et, pour faciliter celui-ci, ils avaient fait nommer à la présidence de l'Assemblée nationale un radical, Midderigh. En échange et dans une lettre de la même époque, l'agent de Barras rappelait à Fokker qu'il recevrait avec reconnaissance les « marques d'amitié et de gratitude » que méritaient ses services. Allusion claire à une récompense qu'il pense avoir gagnée et qui lui a été en quelque sorte promise par Eberstein. Ducange note du reste à plusieurs reprises ses soucis financiers, manière de dire qu'une rétribution serait la bienvenue. Ainsi, le 29 janvier, quelques jours après le coup d'État, il écrit au directeur Van Langen : « Mes moyens sont à sec à peu près, mais peu m'importe au fond, je ne pense pas à cela, de plus, il n'y aurait pas de mérite à faire un peu de bien, s'il n'était le fruit de quelques sacrifices ". En février, il exige deux cent cinquante florins; on lui en remet par la suite douze cents. Ce qu'il a précisément obtenu, cela demeure un mystère, mais le fait est que Eyckenbroek a reçu des lettres de change d'une valeur de 246923 florins, dont à n'en pas douter une partie a servi à financer le coup d'État du 22 janvier et ceux qui l'ont préparé et soutenu' ${ }^{1 \mathrm{x}}$.

Quels ont donc été les services de l'agent de Barras pour mériter des marques d'amitié, si ce n'est qu'il est devenu l'intermédiaire incontournable entre les radicaux et Delacroix et qu'il a tout fait pour que celui-ci collabore avec ceux-là, quitte à minorer les différences entre leurs priorités et

(17) Ce que confirment, si on veut bien les prendre pour argent comptant, les propos de Van Langen : ainsi. Delacroix aurait voulu imposer aux radicaux : l'unité des finances et l'unité parfaite tant audedans qu'au dehors ; l'exclusion des Orangistes des fonctions publiques; l'exclusion du suffrage des adversaires de la Constitution ; la séparation absolue de l'Église et de l'Etat et la confiscation de tous les biens des émigrés. Dans les premiers projets constitutionnels de 1796 et de 1797 , la répression des adversaires était beaucoup plus limitée. Mais Ockerse, lui aussi memhre du comité de constitution, aurait souhaité des mesures plus sévères - notamment un « serment plus sćvère ». DF. Gou, 1798, I, p. XXXVI et 1798, II, p. 322.

(18) Authentique bijlagen tot de geheurnenissen van den 12 juni 1798, Leyde, 1798, pièces 3-7, p. 6 . GS, II, p. 7 (sur une lettre de change de 100000 florins, tirée par Ducange, ce qui fait beaucoup plus que les cent ou les mille ducats promis - respectivement 500 et 5000 florins). 
celles de la France ? Il fait plus. Il laisse traîner les traductions et cherche à gagner du temps. Ainsi quand, le 31 janvier, Delacroix envoie à Talleyrand le décret de l'Assemblée sur les bases du pacte social, il n'a toujours pas la traduction. Mais convaincu comme il est que les patriotes sont de bonne foi et que leurs principes diffèrent peu de ceux de Paris, Delacroix affirme que cette traduction serait inutile " attendu que le dispositif du décret est textuellement semblable aux articles de l'espèce de symbole que les représentants avaient signé avant le 22 janvier $"{ }^{19}$. Non seulement Delacroix s'attend à ce que la commission de constitution demeure fidèle à ces points constitutionnels convenus, mais aussi à ce qu'elle prenne en considération le projet, réalisé par Daunou - confié à Vreede le 27 janvier seulement, et dont il ne sera plus question jusqu'au 5 mars suivant. Pour le reste, le ministre s'en remet à Ducange. Or, celui-ci invoque à plusieurs reprises des prétextes pour retarder la remise des traductions. Le $1^{\text {er }}$ février, il n'a encore rien traduit ; le 8 , il envoie les cahiers - le texte constitutionnel tenu par Ockerse - au ministre pour les lui redemander le lendemain ; le 12, les cahiers sont encore et toujours en sa possession, afin d'être traduits. Faute de temps, Ducange communique de temps à autre une version littérale, sans la mettre au net ou la corriger. De là à savoir si elle est définitive, il y a un pas que nous ne nous risquerons pas à franchir. Ces cahiers du reste ne sont pas toujours en sa possession. Ils passent de main en main, tantôt pour être corrigés par Van Leeuwen, tantôt par Ockerse, de sorte que Ducange ne peut avancer son ouvrage. Il les reçoit ainsi le 14 février mais doit les retourner le jour même à Ockerse, afin que ce dernier les fasse imprimer. Et c'est ce jour-là sculement que Delacroix reçoit les différents documents rédigés par la commission, dont tous ne sont pas forcément traduits. Le lendemain, le ministre écrit pourtant à Talleyrand que ce « qu'il a vu est conforme pour le sens aux bases adoptées ", bien que l'ordre et la rédaction ne soient pas tout à fait identiques. Les 17 et 18 février, le traducteur se fait à nouveau attendre. Il n'a pas eu le temps de travailler. Pis, son domestique a jeté par mégarde la copie traduite et l'original hollandais. Il a dû se procurer un nouvel original et se remettre à la tâche ${ }^{20}$.

Pendant ce temps, Van Langen tentait de convaincre Ducange de la nécessité de modifier plusieurs articles et d'accepter notamment des " élections populaires ", et ce dernier invitait Delacroix à accorder aux Bataves « tout ce qui sera possible, sans s'écarter des principes signés ». Or, fidèles à leurs idées, les radicaux ont décidé de ne pas partager en deux le pouvoir législatif et ils invoquent le rejet de la constitution de 1797 pour prôner ce

(19) C'est nous qui soulignons. DE GoU, 1798, II, p. 380. L' « espèce de symbole »était donc bien les points constitutionnels convenus, approuvés par 49 députés. Ockerse et Delacroix emploient explicitement ce terme, tandis que Ducange utilise un des premicrs l'expression " point convenu ".

(20) Ces lettres ont donc été publiécs par DE Gou (voir note 8). On les trouve aussi aux AN, AFIII-70, dossicrs 283 et 284 . 
qui leur semble indispensable à leur pays : un argument récurrent notamment chez Ockerse, qui plaide en faveur d'une influence directe du peuple - un peuple qui aurait "plus besoin d'éperon que de bride». Ce qui permet à Vreede et à Van Langen de jouer les conciliateurs et d'imposer à Delacroix la séparation après-coup du corps législatif, que n'ose rejeter le ministre, de peur d'éterniser les discussions. Le 22 février suivant, Delacroix affirme à Talleyrand qu'à quelques objets près, la commission ne s'écarte pas des bases adoptées. Pourtant, le 23, Ducange n'a pas encore reçu les titres 4,5 et 6 . Ce qu'il a pour l'instant, c'est un abrégé des articles du titre 4, rédigé par le pasteur radical Konijnenburg. Or, ce dernier aurait par trop bridé le pouvoir exécutif et détruit la majesté du législatif. Qu'à cela ne tienne ! Ockerse a promis de rajouter ce qui est omis dans un Règlement, lequel évidemment n'est pas encore disponible ${ }^{21}$. Pendant que Ducange traduit ces nouveaux titres, Delacroix formule ses observations sur ccux qu'il a en sa possession et les renvoie à la commission. Le traducteur a pour tactique de souligner tel ou tel accroc aux principes convenus, afin de minorer les différends.

La tactique doit avoir eu des effets, puisque ce même jour, interrogé par Delacroix sur ses activités de la veille, Ducange avoue que Van Langen l'avait convoqué pour lui proposer le poste de chef du bureau de la direction des gazettes et de l'esprit public - ce qu'il semble vouloir accepter ${ }^{22}$. Promesse de poste bien rémunéré ; conciliabules avec les chefs radicaux ; offres de services aux uns et aux autres, correspondance affable, voire amicale, mais aussi discrètes remises d'argent : Ducange a servi les intérêts bataves au même titre que les siens, ce qui pourrait expliquer les dépenses inconsidérées de Van Langen, lequel devra en répondre après le coup d'État du 12 juin. Les directeurs Fijnje et Van Langen seront en effet inculpés pour avoir inconsidérément gaspillé les 450000 florins qui leur avaient été confiés pour les dépenses de l'État. Ce qu'il en est au juste, personne ne le saura ${ }^{23}$. Ni Fijnje, ni Van Langen ne dénonceront les bénéficiaires de cette ténébreuse affaire. Prudente, la France s'entremettra en effet pour qu'ils soient libérés, afin que ne soient pas rendus publics les aveux - éventuels - des inculpés. Outre Ducange, Eberstein et Eykenbroek, qui sait combien de diplomates, ministres ou directeurs français n'avaient pas profité des générosités du gouvernement du 22 janvier? Le bouc émissaire

(21) Les règlements ont été rédigés ultérieurement et Delacroix ne semble pas les avoir envoyés à Paris. Larticle sur la révision par le peuple notamment se trouve dans les règlements. Voir les diverses correspondances dans DE GOU, 1798, II, p. 403-432.

(22) Voir le mémoire de Blauw du 10 décembre 1799. GS, 1I, p. 633-673 : " On ébranla même Ducange, parce qu'on lui fit entrevoir une fortune quelconque, accompagnée d'une véritable gloire ". S'il faut lire le texte avec prudence et ne pas ajouter foi à tout ce qui y est avancé, on peut malgré tout le comparer avec le mémoire de Van Langen ou de Fijnje et d'autres documents et en tirer quelques conclusions fiables.

(23) Authentique Bijlagen tot de gebeurtenissen van den 12 juni 1798, op. cit., pièces $n^{\circ} 3,4$ et 7 . Et Nieuwe Nederlandsche Jaerboeken, 1798, II, p. 508-513. 
dans l'affaire, tant en France qu'en Hollande, ce sera tout simplement Brahaïn Ducange ${ }^{24}$.

En attendant, le temps presse. Le 6 mars, le projet doit être présenté à l'Assemblée et il faut le faire imprimer incessamment - Ockerse dixit. Le 14 mars suivant, l'Assemblée est invitée à en discuter. Le 17, elle s'accorde pour l'accepter. Le 18, le projet complet n'est toujours pas entre les mains de Delacroix. Il est pourtant dès lors imprimé et accepté sans que le ministre en ait vérifié la dernière version ${ }^{25}$. C'est aussi ce que suggère un écrit anonyme envoyé au Directoire français, qui prétend que Delacroix ignore tout des détails et des principes de la Constitution batave - ce qui est manifestement faux mais non dépourvu d'une part de véritézh.

Rappelé en juin par le Directoire parisien, Delacroix avouera que la méconnaissance de la langue ne lui a pas permis de s'instruire par luimême de toutes les finesses du texte constitutionnel et surtout de vérifier que toutes ses observations avaient bel et bien été intégrées. Qui plus est, si, sur certains articles il s'est montré intraitable, sur d'autres, auxquels les Bataves tenaient dur comme fer, il a fini par céder. Qu'il n'ait pas non plus réussi à imposer le projet Daunou, précise le ministre, "cela viendrait de ce que le travail de la commission de Constitution était très avancé " et il n'a pas « cru devoir exiger d'Ockerse qu'il sacrifie ses travaux, d'autant moins que le temps pressait et que les points sur lesquels Bataves et Français différaient n'étaient pas essentiels ${ }^{27}$. En fait, il a pensé qu'il suffisait de suivre l'esprit, et non la lettre, de ses instructions. Et quand il s'avèrera que le projet définitif diffère notablement de celui qu'il a eu sous les yeux, Delacroix l'imputera naïvement à l'inadvertance des copistes. À aucun moment, il ne soupçonne les radicaux. Nulle part, il ne mentionne les manceuvres de Ducange. Ce dernier a joué son double jeu en maître consommé. Mais à la nouvelle de son expulsion décrétée fin mai 1798 par le Directoire français, il se sent assez menacé pour s'enfuir en toute hâte et se réfugier à Wesel, en Allemagne, où le rejoindront bientôt plusieurs victimes du 12 juin. Le fin mot de l'affaire, c'est tout simplement que Delacroix fut bel et bien berné, à la fois par Ducange et par les « ultra-révolutionnaires ».

Inversement, les radicaux au pouvoir ont eu plus d'une fois par trop tendance à suivre les idées malencontreuses d'un ministre du Directoire

(24) Tous les témoignages concordent pour confirmer que Barras en tout cas a bien reçu des pots de vin : outre de l'argent, des draps et des eaux de vie qui provenaient des fabriques de Vreede et de Eykenbroek. Van Langen écrit aussi que le club d'Amsterdam (de Uitkijk) avait promis 800000 florins à ceux qui aideraient les radicaux. Eykenbroek en aurait ainsi reçu plus de 200000 à remettre à Barras ; le reste serait payé en marchandises. GS, II, p. 594-619. Ouant à la responsabilité de Ducange, elle apparaissait évidente à la presse politique de l'époque (De Constitutionele Vlieg ou La Mouche constitutionnelle), qui ironisait sur ses intrigues et à Dacndels, qui l'attaque lors d'un dîner. Voir plus loin. De Gou, 1798, II, p. 463.

(25) Il va de soi que Delacroix n'ose pas l'avouer à Talleyrand. Et ce sont des écrits anonymes, où il y a à prendre et à laisser qui dévoilent la manouvre. Cés lettres tentent de déconsidérer à Paris le gouvernement radical. Di: GoU, 1798, II, p. 448-449, notes 30 et 31 ct p. $475-481$. AN, AF-III-70, dossier 284 ..

(26) DE GOU, 1798 , II, p. 447-449.

(27) Ihid., p. $475-476$ et p. 480 . 
pas aussi modéré qu'on a voulu le croire. Puisque c'est à lui que sont dues l'idée d'épurer les assemblées primaires, mais aussi celle de la prorogation du corps législatif et du directoire exécutif, tandis qu'il aurait souhaité envoyer aux « îles » les fédéralistes et les modérés. C'est ce dont se plaint le secrétaire de la légation française, Champigny-Aubin, quand il évoque les " conciliabules secrets de Vreede, Fijnje et Delacroix ", d'où lui-même était bien sûr exclu. Et il note que «certains disent que c'est Delacroix qui porte ce directoire aux mesures tyranniques qu'on lui reproche ${ }^{2 k}$. Quoi qu'il en soit, grâce aux intrigues de modérés ou de légalistes et sans doute aussi en raison de la situation en France suite aux élections de floréal an VI, le Directoire parisien se désolidarise bientôt du gouvernement dirigé par Vreede, rappelle son ministre et soutient le général Daendels, si bien que le 12 juin suivant les radicaux sont à leur tour renversés au profit d'unitaristes légalistes ou libéraux ${ }^{24}$.

\section{La chute des radicaux}

Reste à comprendre les raisons profondes de la chute des radicaux et du rappel de Delacroix ${ }^{311}$, car les arguments invoqués par les hommes du 12 juin sont aussi peu crédibles que ceux des Thermidoriens à l'endroit de leurs prédécesseurs. Pour mieux appréhender ce second mystère, force est de revenir sur les quelques mois qui séparent le 22 janvier du 12 juin suivant et de rechercher ce qui a pu jouer un rôle déterminant.

Avant même l'acceptation de la constitution, dès mars 1798, plusieurs problèmes troublent l'harmonie nationale qu'attendait le régime nouveau. À Amsterdam, la Société pour l'Unité et l'Indivisibilité - pourtant radicale - désapprouve le licenciement de la municipalité et son remplacement par des personnes nommées - et non élues - dont certaines n'auraient pas le profil de l'emploi ${ }^{3 !}$. Argument qui deviendra récurrent et

(28) AE, vol. 599. Correspondance politique. Hollande. Lettre du 14 prairial an VI.

(29) Au licu de parler d'unitaristes modérés, je préfère le termc de « légalistes » ou de « pragmatiques ", car parmi ces prétendus modérés figurent des hommes comme Gogel et Wiselius, qui conservent des aspirations réellement démocratiques, mais désapprouvent l'État d'exception qu'est devenu le régimc de Vreede et consorts. Eux veulent introduire la Constitution et ceuvrer en toute légalité à la prospérité du pays. Quoi qu'il en soit, les catégories politiques sont ici différentes de celles de la France; de méme les haines politiques ne sont pas insurmontables. Sur un premier essai sur le sujet, "Napoléon et les élites patriotes bataves : un méme combat ? ", dans Napoleon et l'Europe, éd. Thierry LENTz, Paris, Fayard, 2005, p. 226-257.

(30) Delacroix avait rempli sa mission mieux encore que n'aurait osé le penser le directoire exécutif, puisqu'il avait également réussi à conclure un traité secret avec les radicaux, favorables à la France. Le seul avantage que devait en retirer la République batave était une réduction des troupes de sćjour sur leur territoire.

(31) Les sociétés populaires étaicnt surtout hostiles à l'article 18 de la déclaration, qui limitait le droit de réunion politique. Des agents du directoire batave ont tout fait pour convaincre ces sociétés de ne pas retarder plus l'entréc dans l'ère constitutionnelle. De là leur revirement. DF Gou, 1798, II, p. 439 et p. 444-446. GS, 1798, Il, p. 785-786; $796 ; 785-786$. Sur ces problèmes, Annie Jourdan, « Amsterdam en révolution. Un jacobinisme batave ? ", Working Paper, University of Amsterdam. 2006. 
qu'invoquera également le ministre des Finances Gogel à l'endroit de Delacroix. La Société, il est vrai, manifeste par la suite son approbation et le 5 avril envoie une adresse signée par 292 personnes pour réclamer des mesures révolutionnaires et des destitutions en masse. Car outre les épurations des assemblées primaires, il est prévu de licencier tous les fonctionnaires orangistes, fédéralistes ou ceux qui sont ennemis du gouvernement nouveau et qui refusent de prêter le serment de haine, imposé par la constitution. Cette mesure en revanche est populaire et dans le pays tout entier, les patriotes, et notamment les ex-réfugiés, se pressent dans leur municipalité respective pour exiger les places remplies par les ennemis de la révolution. La situation est évidemment sérieuse dans les grandes villes, telle Amsterdam, où la municipalité compte 3000 places dont un nombre important à redistribuer.

Parallèlement ont donc lieu les épurations des assemblées primaires. Des abus sont commis. Le directoire exécutif - que le corps législatif provisoire a prorogé le 17 mars - cherche à corriger ces erreurs et crée une commission qui en est spécialement chargée. Elle est confiée au département du ministère de la Police, lequel ne semble pas avoir fait des excès de zèle. C'est du moins ce que lui reprochera l'ex-directeur Wybo Fijnje dans son mémoire de justification ${ }^{32}$. Qui plus est, après l'acceptation de la constitution, il est prévu d'ouvrir les registres à quiconque n'est pas concerné par les articles 13 et 15 du texte constitutionnel - qui excluent certaines catégories politiques du suffrage. Les épurations ne sont donc que temporaires et ne mènent pas non plus à des poursuites judiciaires. Elles n'en mécontentent pas moins ccux qui les jugent injustes à leur égard, ainsi qu'en témoigne le flot de pétitions et d'adresses qui inondent l'Assemblée.

Ce sont là les premiers mécontentements, mais il en est d'autres, sans doute plus graves. Notamment ceux de plusieurs complices du coup d'État, qui jugent ne pas avoir été récompensés dignement. Le général Daendels revendique ainsi des places pour son neveu et ses amis; Joubert fulmine de n'avoir reçu que deux chevaux en guise de récompense ; il s'attendait à mieux et demande un supplément de 1000 florins par mois ; l'amiral de Winter, de retour de captivité, exige de recevoir un arriéré de six mois de salaire, là où l'ambassadeur à Paris, Caspar Meyer, réclame 80000 florins de rémunération pour ses bons services. À ces exigences multiples, qui sont peu souvent suivies d'effets et qui mènent donc à des ressentiments s'ajoutent les rivalités entre ministres eu égard à leur résidence ou à leur personnel. Tout cela n'est rien en regard des 800000 florins que le gouvernement batave doit payer à Paris ou de la lettre de change de 100000 florins qu'aurait encaissée Ducange. Delacroix lui-même aurait

(32) Sur ce mémoire retrouvé parmi les papiers de Galdi, l'article à paraitre de M.VAGHI. 
reçu 10000 florins pour avoir permis de réduire les troupes françaises en terre batave. Des sommes qui ne sont pas même disponibles dans les caisses de l'État. Le gouvernement les a trouvées vides et ne sait pas très bien comment les remplir. Imposer le peuple qui ne l'est que trop ne lui paraît pas une bonne solution : ce serait "se dépopulariser ", alors que l'opinion doit encore accepter la constitution. En appeler aux banquiers, aux négociants, aux marchands ou aux régents, ainsi qu'il en allait d'ordinaire, cela ne se peut, puisque pour une grande part, ils viennent d'être interdits de suffrage. Van Langen et Fijnje, deux des directeurs, auront beau frapper chez Van Staphorst ou chez Collot d'Escury ${ }^{33}$, ce sera en vain. Pourtant le banquier patriote Van Staphorst connaît très bien les difficultés financières rencontrées par la république batave. En 1795, il avait lui-même été chargé de stimuler les prêts et appris ce qu'il en coûtait à la révolution. Pour obtenir les sommes nécessaires afin de paycr les échéances à la France, il avait dû faire rapporter plusieurs décrets qui visaient les régents orangistes ou les exportations de capitaux. Bref, aux Pays-Bas, le monde de la finance avait le pouvoir d'orienter la politique révolutionnaire dans un sens qui la trahissait. S'y refuser, c'était acculer le pays à la ruine et à la perte de l'indépendance $^{34}$. Or, le gouvernement de janvier 1798 a la maladresse de s'aliéner ces hommes sous plus d'un point ${ }^{35}$.

Devant la pénurie du gouvernement et les revendications populaires, le $\mathbf{3 0}$ mars suivant, le corps législatif décide en effet de poursuivre les régents de 1787 ou leurs héritiers et de les spolier afin d'indemniser les patriotes lésés lors de la restauration. Le remède est en somme pire que le mal, et, si le décret satisfait les radicaux populaires qui demandaient depuis janvier 1795 des mesures répressives contre l'ancien gouvernement et des indemnités en faveur des vrais patriotes, les " hommes de bien " crient au scandale et à la Terreur. Lon comprendra que, dans ces conditions, rares soient les régents, les banquiers ou les négociants qui désirent contribuer au paiement des quarante millions à payer d'ici peu à la France. Ce décret,

(33) Rohert Collot d'Escury, receveur général des ressources maritimes de 1785 à 1805 , était le gendre de Bicker, régent influent et ci-devant patriote réfugié en France. Or, Bicker avait été interné par le gouvernement du 22 janvier 1798, voir J. RosenDAAL, op. cit., p. 24-25, 75-76, 582-583 et S. SCHAMA, op. cit., p. 309.

(34) Joanna Fritschy, De patriotten en de financiën van de Bataafse Republiek, La Haye, 1988, p. 211-217. La France menaçait de mettre fin à l'indépendance hatave si l'alliée ne payait pas les 100 millions exigés. Or, la Hollande ne pouvait trouver les sommes nécessaires ailleurs que chez les financiers, banquiers, négociants, qui n'étaient pas forcément patriotes et surtout pas du parti radical de Vreede. Véritable dilemme ! Sur les rapports entre gouvernants et financiers, Marjolein 'T HART \& alii, A Financial History of the Netherlands, Cambridge, Cambridge University Press, 1997.

(35) Voir le mémoire de Van Langen, GS, II, p. 594-619. Van Langen avoue qu'ils ont emprunté un million à l'ancienne compagnie des Indes orientales, elle aussi endettée et épuisée par ailleurs - ce qui n'a pu qualiéner du gouvernement radical, Wiselius, qui était membre de la dite compagnie, aux côtés de Fijnje. Tous deux étaient du reste amis jusqu'au 22 janvier et le redeviendront en 1799. Christina KroesLigtenberg, Dr. Wybo Fijnje (1750-1809), Assen, 1957, p. 190-204. 
publié le 31 mars $^{36}$, va opposer par ailleurs le directoire exécutif au corps législatif et créer un nouveau sujet de mécontentement au sein même du gouvernement. Le 11 avril, les directeurs publient ainsi une lettre de désaveu, s'inquiètent des injustices à venir et mentionnent les conséquences néfastes qui vont découler de telle mesure : «Mal appliqué, il conduira à la banqueroute des fabricants ; à la fermeture des entrepôts ; à l'arrêt du commerce, sans oublier les effets néfastes sur le crédit, comme l'a démontré sa chute lors de la publication dudit décret ". Ils invitent donc le corps législatif à revenir sur une décision qui ne saurait que mener la patrie à la ruine $^{37}$. La première conséquence du décret avait aussitôt provoqué une chute des cours ; le retrait du décret entraîne la hausse. Mais les législateurs ne s'avouent pas vaincus. Le 4 mai sont encore à l'ordre du jour à la tribune de l'Assemblée le problème des indemnités et la spoliation des adversaires de la révolution. Ce serait à l'ancien gouvernement (de 1787) de payer les dépenses de la République. Ses membres et leurs amis ou créditeurs, on s'en doute, n'en sont pas convaincus, d'autant moins qu'ils ont déjà prêté de grosses sommes en 1795. Blauw, qui se souvient avoir prônć de telles répressions au début de la révolution, lesquelles, selon lui, auraient alors eu l'avantage de réunir tous les patriotes, dénonce l'absurdité d'une mesure prise après trois ans de troubles, qui aurait "frappé presque toute la nation " et serait "inexécutable dans les formes". Exécutable ou non, elle eût mené à une fuite d'hommes et de capitaux et bouleversé gravement la petite république.

Le 15 mai suivant, l'Assemblée nationale examine par ailleurs comment mettre sous séquestre les biens des émigrés et demande que soit recherché qui en sera passible ${ }^{3 k}$. Ces décrets ou projets de décrets n'ont rien pour combler des hommes avides de stabilité et désirant recouvrer un crédit endommagé par les troubles révolutionnaires. Et de fait, les adversaires du gouvernement radical vont s'accroître de tous ceux qui se défient des chocs et des grands bouleversements. Il leur paraît insensé que la classe des régents soit considérée comme " une personne morale ", censée être punie en bloc, que les individus parmi eux aient ou non contribué à la répression des patriotes. Ce serait désagréger la république ; tomber dans le déshonneur ; ruiner le peu de crédit qui subsiste. Ces problèmes vont donc aliéner des radicaux unitaires plusieurs de leurs anciens amis ou sympathisants. À la tête de ces adversaires nouveaux se trouve le ministre

(36) Nieuwe Nederlandsche Jaerboeken, 1798, I, p. 176-178. Dagverhaal, 1798, en date des 30 et 31 mars 1798. Oue je sache, aucun historien n'a noté ce décret et l'effet désastrcux qu'jl a eu auprès des " hommes de hien ", seuls aptes à financer l'indépendance batave.

(37) NNJB, 1798, I, p. 229-233. Gogel lui aussi aurait souhaité intervenir et interdirc les investissements à l'étranger et la fuite de capitaux, mais il se refusait à ce que l'on terrifie les régents et leur descendance, justement pour éviter la fuite de capitaux. GS, II, p. 785.

(38) Dagverhaal, 1798, no.12, p. 91. AN, AF-III-70, 2. 
des finances, Isaac Gogel, qui redoute que le régime de 1798 n'aboutisse à " la chute du triste reste du crédit national ; [à] la perte du commerce déjà trop ruiné ; celle de toute confiance pour les lettres de change chez l'étranger; l'extinction de l'enthousiasme ct de l'esprit républicain, l'émigration et l'impossibilité de trouver des ressources financières dans les bourses des citoyens, dont le cœur déteste le gouvernement qui en a besoin $\aleph^{39}$. La crainte était sensée. Tous ceux qui avaient quelques biens ne se retrouvaient plus dans une république "inconstitutionnelle ", qui adoptait peu à peu des traits " jacobins ». D'après l'agent consulaire Pichon, nombre d'entre eux songeaient dès lors à s'expatrier, eux et leurs portefeuilles.

Le 4 mai a été proclamé un second décret qui a fait bondir la république tout entière. Sur les " bons " conseils de Delacroix, le corps législatif a décidé d'imiter la France et de se proroger pour les deux tiers. Mais contrairement à la République mère qui l'avait porté à l'approbation populaire, le décret est ni plus ni moins imposé par l'Assemblée, ce qui le rend plus inique encore. Lindignation qui accueille cette décision est plus violente que celle relative au décret du 31 mars, car elle touche tous les citoyens. Si la spoliation des orangistes et des régents était depuis longtemps à l'ordre du jour et répondait aux attentes des patriotes populaires et des ultra-révolutionnaires, priver tous les citoyens bataves de leur droit d'élire leurs représentants, c'était là une atteinte à la Constitution (tout juste sortie de son arche), qui allait rallier contre le gouvernement tous les mécontents de quelque parti ou classe qu'ils soient. Aussitôt se constitue donc une coalition d'hommes de talent et d'argent mais aussi de patriotes sincères, déçus par l'illégalisme de la mesure.

À en croire le secrétaire de la légation française, depuis le 22 janvier, le directoire batave s'obstinerait à prendre des mesures " ultra-révolutionnaires ». Les directeurs influents, Pieter Vreede et Wybo Fijnje surtout, se monteraient fortement l'imagination et auraient dit " qu'ils se feraient hacher un pied plutôt que de reculer [...] et qu'ils n'obéiraient en aucun cas au gouvernement français ". Le 18 prairial (6 juin), pourtant, le directoire batave se rend à l'évidence et décide de soumettre le décret sur la prorogation du corps législatif au suffrage du peuple : «Il veut rapprocher les esprits... mais en a-t-il encore les moyens ? ", commente Champigny Aubin $^{\text {th}}$. C'est trop tard en vérité. Les radicaux se sont aliéné non seule-

(39) GS, II, p. 762 (lettre à Delacroix, non datée). À cette époque, Gogel est en lutte contre Amsterdam, qui se refuse à ce que deux rapports soient établis sur les finances de la ville : un par la municipalité ; l'autre par la banque de prèt. La municipalité " régénérée " est vexée par ce qu'elle voit comme une défiance de la part du ministre et comme une enfreinte à sa souveraineté. Aux problèmes entre directoire exéculif et ministres s’ajoutent donc ccux entre les ministres et les municipalités, qui refusent de perdre leurs prérogatives et de n'être plus qu'administratives. Sur le problème, la thèse imprimée de Thomas POEL. The Democratic Paradox. Dutch revolutionan struggles over Democratisation and Centralisation (17801813), s.e.,Utrecht, 2007.

(40) AE, 599, lettres du 14 au 18 prairial an VI. 
ment leurs compatriotes, mais encore le gouvernement français et Joubert, le général en chef des troupes franco-bataves.

Le général Joubert n'a rien fait pour contrer le coup d'État du 12 juin. Bien au contraire. C'est à lui qu'est due l'impunité du général Daendels, passible de poursuites pour avoir déserté son poste. Au mois de mai 1798, ce dernier en effet avait insulté Delacroix et Ducange lors d'un dîner officiel et était ensuite parti se réfugier en France, à l'abri des poursuites qu'allait entreprendre contre lui le directoire batave. Réconforté par l'accueil qui lui fut fait à Paris, il est revenu le 10 juin dans l'espoir de mettre fin à un régime qui lui était trop peu reconnaissant et qui, de surcroît, menaçait de s'en prendre à sa liberté. Que Joubert ait laissé faire, c'est en somme ce que lui avait suggéré Talleyrand ${ }^{41}$. Du coup, il n'est pas inutile de se demander si les radicaux unitaristes n'avaient pas non plus mécontenté leur allié français.

Qu'ils se soient aliéné Joubert, cela ne fait aucun doute. Malgré une rémunération mensuelle de 1000 florins, le général était insatisfait. D'une part, les directeurs refusaient de lui permettre de visiter les arsenaux ou casernes du pays. D'autre part, ils venaient d'augmenter la garde de La Haye sans le consulter. Or, Joubert se voit comme le commandant en chef des troupes franco-bataves et exige d'être impliqué dans toute mesure relative aux armées. Et puis, il y a les conflits interminables et incessants à propos du port de Flessingue. Les Français revendiquent une part des droits de douane qui y sont encaissés. Ils y ont même envoyé des douaniers pour s'en occuper, au grand dam du gouvernement batave, qui interprète cette présence comme une violation du territoire national et de sa souveraineté et qui expulse donc les dits douaniers. Le Directoire parisien en était même venu à revendiquer l'entière possession du port, contre une cession de territoires moins importants. Un marché désavantageux pour la République batave laquelle ne veut donc pas en entendre parler. Que dire enfin des corsaires français, qui sèment la zizanie entre les deux républiques ? En 1798, ils exigent un vaisseau rempli de genièvre, appartenant à des Néerlandais que refuse évidemment de leur céder le gouvernement ${ }^{42}$. Vexations, irritations, les heurts entre les deux alliés étaient légion. De là à penser qu'un gouvernement nouveau et plus souple faciliterait les relations avec la République mère, il y a un pas qu'ont sans doute franchi les Français. Contrairement à Delacroix qui soutenait de tout son poids le gouvernement du 22 janvier, le secrétaire de la légation encourageait ce changement et aspirait à un retour sur scène des hommes " sensés ", des

(41) Talleyrand lui écrivait énigmatiquement : "le sort de la Hollande est entre vos mains ". AE, 599. Lettre du 23 prairial an VI.

(42) Sur ces problemes récurrents et irritants, AE, Vol. 598, 599 et 600. 
"vrais républicains ". Il va sans dire que c'est aussi ce que suggéraient les intrigants de tous genres qui affluaient à Paris. Si l'on en croit Blauw, peu élogieux sur le Directoire français, celui-ci n'avait pas les moyens de « rémunérer grassement » ses gouvernants et leurs ministres, lesquels en étaient donc réduits à rechercher ailleurs des ressources. Les radicaux n'avaient pas les moyens de les leur procurer. Dans l'autre camp, celui des unitaristes légalistes ou libéraux, ceux-là même qui cachaient leurs réserves et leurs trésors, peut-être y avait-il plus à gagner. Les intrigants ont dû jouer sur cette corde. Là réside sans doute une part de véritéét. Pour le reste, et ainsi qu'en témoignent les propos susnommés de Champigny Aubin, les radicaux bataves s'étaient manifestement montrés trop peu conciliants et se refusaient à obéir servilement à la France.

À la même date, par ailleurs, s'accumulent par le biais de lettres anonymes adressées à Paris les plaintes contre le gouvernement batave - le taxant d'extrémisme ou d'anarchisme. Au lendemain du décret du 4 mai 1798 , plus que jamais, on parle de mesures arbitraires : ce qui paraissait énergique juste après le coup d'État devient " sévices imposés aux vrais amis de la liberté ». C'est que les commissaires du directoire batave ont commis des excès de zèle, qui irritent les hommes éminents : Wiselius et Schimmelpenninck notamment, qui se sont vus privés du droit de suffrage. Dans les administrations locales, enfin, se seraient introduits des « hommes sans talent ", qui en ont chassé les honnêtes gens ; des "incapables", plus soucicux d'épurations et de places que de mesures positives. Les impôts souffriraient ainsi des retards. Lévolution est telle qu'elle indigne donc le ministre des Finances, Gogel, lequel participera au complot pour renverser les hommes du 22 janvier, avec à ses côtés les autres ministres, et à l'exception de van Kooten, responsable de l'Éducation nationale et ami intime de Valckenaer. Les mesures arbitraires ou inconstitutionnelles qui se succèdent, scandalisent donc les unitaristes légalistes, pragmatiques ou libéraux. Et ils sont nombreux en Hollande. Gogel, Goldberg, Wiselius ${ }^{44}$, mais aussi Blauw et Daendels se désolidarisent du gouvernement et se tournent vers Paris, dans l'espoir de convaincre le Directoire d'accepter un nouveau bouleversement. La coquette somme de trois cent mille florins et la situation en France - le coup d'éclat du 22 floréal - contribuent à amadouer le

(43) Une part du mystère seulement, car Blauw est particulièrement monté contre le directoirc parisien et contre Delacroix. Pour l'autre, ce sont donc les rivalités intérieures et les problèmes avec le port de Flessingue qui ont joué. GS, II, p. 633-693. Blauw par la suite semblerait avoir tenté de sauver les radicaux, mais à force d'intrigues, il s'aliène le Directoire français et le gouvernement du 12 juin. AE, 598, lettre de Daendels à Rouget et lettre de Pichon à Talleyrand du 3 messidor an VI.

(44) Gogel est donc aux finances ; Goldberg est à l'économie et Wiselius à la Compagnie des Indes orientales. Gogel et Wiselius étaient membres du Comité révolutionnaire d'Amsterdam en 1795. Goldberg lui aussi patriote de $1780-1787$, avait ćté nommé membre du gouvernement provisoire de la ville d'Amsterdam. Il sera à lorigine des premières statistiques nécrlandaiscs. 
gouvernement parisien ${ }^{45}$. Avec le soutien de Joubert, Daendels renverse alors ceux qu'il avait mis au pouvoir quelques mois auparavant. Les radicaux prennent à leur tour la route de Huis ten Bosch - ci-devant résidence du stadhouder, où avaient été emprisonnés les modérés et les fédéralistes en janvier 1798, mais aussi des hommes qui tout simplement désapprouvaient le coup d'État : Gevers, Van de Kasteele, Van Hooff ou Hahn, tous unitaristes convaincus. Entre-temps, la Hollande avait une constitution ${ }^{4 h}$ et c'était cela seul qui comptait vraiment pour Paris : faire en sorte que la république sœur ait une existence politique réelle et remplisse ses engagements.

Ce que dévoile plus particulièrement cette étude de détail, c'est que le texte de loi rédigé durant ces quelques mois était donc pour une grande part d'origine batave et qu'il s'est inspiré tout à la fois du manifeste des 43 et des idées consignées par Ockerse vers la même époque, tandis qu'ont été repris plusieurs points auxquels tenait le gouvernement français, à l'initiative de Daunou et de Delacroix - et même si plusieurs d'entre eux ont été revus et corrigés ${ }^{17}$. Pour s'en persuader, il n'est que de lire la déclaration des droits de l'homme et du citoyen, placée en préambule et qui compte huit «principes généraux» et soixante-quatre «principes civils et politiques ", là où les déclarations françaises successives en comprennent dixsept (1791), trente-cinq (1793) et trente et un (1795). Lintitulé lui-même diffère de celui de la France et suggère que les Bataves entendent formuler non une simple profession de foi des législateurs mais les principes à la base du pacte social qui lie le peuple à lui-même. Contrairement au texte français de 1795, où ont disparu les droits dits sociaux, la Constitution de 1798 mentionne amplement les devoirs de la société envers ses membres, non parce qu'elle renoue avec la déclaration jacobine, ainsi que l'avance

(45) La République batave sait trouver de l'argent quand l'enjeu en vaut la peine. Ainsi régents, marchands, négociants et financiers d'Amsterdam et de Rotterdam proposent à l'automne 1798 une grosse somme à Schimmelpenninck. pour qu'il demeure ambassadeur à Paris et continue à défendre leurs intérêts. DE WIT, De strijd tussen aristocratic en democratie in Nederland (1780-1848), Heerlen, 1965, p. 174-177. Amsterdam lui offre 55000 florins ; Rotterdam, 19000 ; l'État lui cn concède 15000 et $180 \%$, de même qu'une rémunération de 25 florins par jour. En ce mois de septembre 1798 , Schimmelpenninck dispose donc de la coquette somme de 107000 florins, auxquels s'en ajoutent par an 45250 (en francs, les sommes doivent être multipliées par deux). GS, II, 475-486.

(46) La Constitution de 1798 est portéc au suffrage du peuple le 23 avril et acceptée par 153913 citoyens actifs et rejetéc par 11 597. DI Gou, 1798, II, p. 456.

(47) Ainsi la division du corps législatif en deux chambres, exigée par la France. Chez les Bataves, elle s'opère après l'élection en bloc des députés; de même par défaut de suffrage direct, les citoyens actifs élaborent une liste sur laquelle vont choisir les électeurs. Sont citoyens actifs tous ceux qui paient une contribution quelconque, à savoir ceux qui gagnent plus de trois cents florins par an, ce qui inclut notamment les ouvriers des chantiers navals. Enfin, le peuple aura le droit de réviser la Constitution. Sur les déclarations élaborées depuis 1795 , voir mon site à l'Université d'Amsterdam. Mon ouvrage à paraître traite en détail cette problématique, mais passe très rapidement sur les intrigues de Ducange \& cie. Cet article est donc le fruit d'études inédites et en constitue un complément. 
Lucien Jaume dans une comparaison superficielle ${ }^{48}$. Le souci d'apporter de l'aide aux pauvres émane d'une tradition protestante autochtone où les communautés religieuses et corporatives possédaient et géraient des institutions très sophistiquées, admirées de l'Europe tout entière. L'État unitaire allait détruire ces corps et se voit donc contraint de remplir leurs tâches. Tout aussi bataves sont la répugnance pour une prédominance de la loi sur les droits et la volonté de conserver au peuple une influence directe sur son gouvernement - par où les radicaux de 1798 sont en vérité plus proches des Américains que des Français ${ }^{49}$. En réalité, dans l'Assemblée batave, seuls deux législateurs s'avèrent de fervents robespierristes : le fédéraliste frison Coert van Beyma et l'unitariste hollandais, Ploos van Amstel. Pour la plupart, les radicaux de quelque mouvance qu'ils soient, se réfèrent de préférence à Rabaut Saint-Étienne ou à Condorcet ${ }^{50}$.

Que la Constitution ne soit ni robespierriste, ni française, c'est ce que révèlent non seulement une étude précise des intrigues menées à partir de janvier 1798 et une analyse des articles déclaratoires et constitutionnels rédigés au lendemain du coup d'État, mais encore le fait que les prétendus (unitaristes) modérés du 12 juin aient conservé le texte et abrogé fort peu de lois ${ }^{5}$. Tout cela démontre enfin que ce ne sont pas tant les principes qui divisaient les unitaristes que les moyens employés pour les mettre en cuvre. De là l'idée qu'aux dirigistes du type Vreede et Fijnje s'opposaient des légalistes, tels que Gogel, Goldberg ou Wiselius. Légalistes non

(48) Lucien Jaume, Les déclarations des droits de l'homme, 1789-1946, Paris, GF-Flammarion, p. 315-317. La méme critique vaut pour la référence à l'Être Suprême, vue dans cet ouvrage comme robespierriste, alors qu'elle est très courante un peu partout en Europe dès les Lumières.

(49) Pensons aux conventions périodiques où le peuple s'exprime sur les lois de l'État auquel il appartient ou bien aux Town Meetings. Gordon WOOD, La création de la République américaine, Bclin, Paris, 1991, p. 376 et p. 704. La constitution de l'an I prévoyait certes des conventions nationales, ainsi que l'avait projeté du reste Condorcet sur l'exemple des États-Unis, mais alors que Condorcet conférait à ces assemblées un pouvoir réel, puisqu'une seule assemblée primaire pouvait mettre en branle le processus, la Constitution de juin 1793 permet une intervention des assemblées primaires seulement après que dans la moitié des départements, plus un, le dixième d'entre elles ont demandé une révision. De méme, elle supprime la censure du peuple, qu'avait prévue ce méme Condorcet. Jacques Godechor, Les Constitutions de la France depuis 1789, Paris, GF-Flammarion, éd. de 1995, p. 91 et Albert Mathiez, Girondins et Montagnards, Paris, 1930, p. 82-108.

(50) Pour mieux comprendre la révolution batave, force est de connaitre celle des patriotes (influencés par l'Amérique et par leur propre tradition républicaine). Ensuite seulement on peut se tourner vers la France : notamment chez Condorcet, souvent cité à la tribune et qui, comme les radicaux, défendait une démocratie "participative ". telle cellc que souhaitait Rohespierre en 1791 . Autres références privilégiées : Rabaut Saint-Étienne qui lui aussi plaidait en faveur du contrat social (en lieu et place dune déclaration) ; Sieyès (entre autres pour son jury constitutionnaire) et Boissy d'Anglas (invoqué par ceux qui craignent la tyrannie d'une assemblée unique).

(51) Mais la loi du 31 mars sera abrogée dès le 25 ou 26 juin, comme contraire aux droits de l'homme et source de déshonneur de la nation batave. La même chose vaut pour celle du 12 avril contre les députés contestataires, également abolie au cours du mois de juin. Jaarboek der Bataafsche Republiek. Amsterdam, 1798, XIII, p. 97-103. 
par culte de la loi, mais par respect de la parole donnée et par crainte d'un déclin plus grave encore de la République ${ }^{\mathrm{s2}}$.

\author{
Annie JoURDAN \\ Université d'Amsterdam \\ European Studies \\ Spuistraat 134, 1012VB-Amsterdam \\ Pays-Bas \\ a.r.m.jourdan@uva.nl
}

(52) Et puis, il fallait compter avec les libéraux du type Van Hooff et Hahn qui désirent protéger les droits des minorités contre la loj de la majorité. Cf. mon article "Les minorités politiques dans la Révolution batave ", à paraître dans les actes du colloque d'Aix. 